\title{
Isolation and characterization of a chalcone isomerase gene promoter from potato cultivars
}

\author{
M. Chen ${ }^{1}$, W.J. Zhu' ${ }^{1}$, X. You ${ }^{2}$, Y.D. Liü, G.M. Kaleri' ${ }^{1}$ and Q. Yang ${ }^{1}$ \\ ${ }^{1}$ College of Life Sciences, Nanjing Agricultural University, Nanjing, China \\ ${ }^{2}$ College of Sciences, Nanjing Agricultural University, Nanjing, China \\ ${ }^{3}$ College of Animal Science and Technology, Nanjing Agricultural University, \\ Nanjing, China \\ Corresponding author: Q. Yang \\ E-mail: chenmincm@njau.edu.cn / qyang19@njau.edu.cn
}

Genet. Mol. Res. 14 (4): 18872-18885 (2015)

Received September 10, 2015

Accepted November 15, 2015

Published December 28, 2015

DOI http://dx.doi.org/10.4238/2015.December.28.37

ABSTRACT. Chalcone isomerase $(\mathrm{CHI})$ is a key enzyme involved in
anthocyanin metabolism. Previous research on $\mathrm{CHI}$ has mainly focused on
CDNA cloning and gene expression. In the current study, the 1425-bp potato
$\mathrm{CHI}$ promoter (PCP) was isolated from four potato cultivars (Heijingang,
Zhongshu 7, Désirée, and Favorita) using PCR and DNA sequencing. The
PCP contained many cis-regulatory elements (CREs) related to anthocyanin
metabolism, tissue specificity, light response, stress, and hormone induction.
Of the PCP CREs identified, 19 were common to those found in the higher
plants examined, based on plant CRE databases. Multiple sequence alignment
showed six single nucleotide variation sites in PCP among the potato cultivars
examined, resulting in changes in the number of CREs connected with tissue
specificity, anthocyanin metabolism, and light response. The 665 -bp PCP
fragments from Favorita and 1425 -bp PCP fragments from Heijingang were
used to construct plant expression vectors, which may be a useful tool for
biological engineering. A transient expression assay demonstrated that the
two PCP fragments from Heijingang could direct the expression of a green 
fluorescent protein gene in onion epidermis and a $\beta$-glucuronidase gene in all potato tuber tissues with different colors, suggesting that the single nucleotide variation in the PCP did not affect its activity, and that silencing of the $\mathrm{CHI}$ gene in Favorita may be attributed to other regulatory factors.

Key words: Potato; $\mathrm{CHI}$ promoter; Cis-regulatory elements; Single nucleotide variation; Activity

\section{INTRODUCTION}

Anthocyanins are a type of flavonoid, and they play a role in many biological processes, such as plant color formation (Koes et al., 2005), pollen development, UV protection (Bieza and Lois, 2001), and stress response (McKhann and Hirsch , 1994). Moreover, anthocyanins have an extensive medicinal value to human health, including inhibition of the proliferation of two types of human cancer cells (HL-60 promyelocytic leukaemia and HT-29 colorectal adenocarcinoma) in a dose-dependent manner (Netzel et al., 2007), significant reduction in tert-butyl hydroperoxide-induced oxidative injury, and the proliferation index of intestinal adenoma (Cai et al., 2010; Hwang et al., 2011). Therefore, anthocyanins have received increasing attention over the past decade.

Chalcone isomerase $(\mathrm{CHI})$ is an important enzyme in the phenylalanine metabolism pathway, which controls tissue-specific flavonoid biosynthesis in plants. The $\mathrm{CHI}$ gene was first isolated from pea using an antibody technique (Mehdy and Lamb, 1987). To date, cDNA sequences encoding $\mathrm{CHI}$ have been cloned from many higher plants, such as Petunia hybrida (van Tunen et al., 1988), Zea mays (Grotewold and Peterson, 1994), Saussurea medusa (Li et al., 2006), and Arachis hypogaea (Liu et al., 2015). CHI shows high expression levels in plant tissues that contain abundant flavonoids or anthocyanins, and overexpression of the $\mathrm{CHI}$ gene has been shown to increase the accumulation of flavonoids in transgenic tomato fruits and the hull of mutant rice (Muir et al., 2001; Wang et al., 2010; Hong et al., 2012). In contrast, CHI suppression by RNAi has been reported to reduce pigmentation and change flavonoid components in flower petals of tobacco (Nishihara et al., 2005).

Promoter sequences control transcription of genes in conjunction with RNA polymerase and transcription factors. Many important cis-regulatory elements (CREs) are located within promoter sequences, and these are the binding sites for corresponding transcription factors. Analysis of promoter sequences can further our understanding of gene expression and regulation. To date, limited research has been undertaken on the $\mathrm{CHI}$ promoter, which was first isolated from the Petunia hybrida inbred line V30 in 1989 (van Tunen et al., 1989).

Potato is an important crop worldwide and its tubers are unique parts that are used in our diet. Interest in potatoes with pigmented flesh has grown, partly because of increased antioxidant content in pigmented tubers with abundant anthocyanins (Naito et al., 1998; Rodriguez-Saona et al., 1998; Eichhorn and Winterhalter, 2005). In previous studies, we cloned anthocyanin structural genes, including CHS, F3H, DFR, ANS, and 3GT from wild potato (Lu and Yang, 2006; Wang et al., 2011), but studies on their promoters remains elusive. In this study, we isolated $\mathrm{CH}$ / promoters from 4 potato cultivars with different colored flesh, compared their structures, and analyzed their activity by assessing transient expression of a green fluorescent protein gene (GFP) and a $\beta$-glucuronidase gene (GUS). 


\section{MATERIAL AND METHODS}

\section{Plant material and growth conditions}

The potato cultivars used in this study were Zhongshu 7 (yellow skin and white flesh), Hui-2 (yellow skin and yellow flesh), HQ (red skin and yellow flesh), Ziyun 1 (purple skin and purple flesh), Heijingang (purple skin and purple flesh), Favorita (yellow skin and yellow flesh), and Désirée (red skin and yellow flesh). These cultivars were grown under natural light and temperature in spring in Nanjing, China. Genomic DNA was extracted from the leaves of the potato plants and 60-day-old tubers were harvested for RT-PCR analysis and transient expression assay.

\section{RNA extraction and RT-PCR analysis}

Total RNA was isolated from the peeled flesh of the seven cultivars using Trizol reagent (Invitrogen, USA), according to the manufacturer protocol. RNA was then converted into cDNA using random primers and reverse transcriptase (Promega, USA), following treatment with DNase (Promega). To analyze expression of the $\mathrm{CHI}$ gene, two primers - were designed, $\mathrm{CHI}-\mathrm{F}$ and $\mathrm{CHI}-\mathrm{R}$, and primers $18 \mathrm{~S}-\mathrm{F}$ and $18 \mathrm{~S}-\mathrm{R}$ were used to amplify potato $18 \mathrm{~S}$ ribosomal RNA, as an internal control (Table 1). All PCR reactions were performed under the following conditions: 5 min at $94^{\circ} \mathrm{C}$, followed by 35 cycles of $40 \mathrm{~s}$ at $94^{\circ} \mathrm{C}, 40 \mathrm{~s}$ at $54^{\circ} \mathrm{C}$, and $1 \mathrm{~min}$ at $72^{\circ} \mathrm{C}$, with a final extension step for $10 \mathrm{~min}$ at $72^{\circ} \mathrm{C}$.

\section{Isolation of the $\mathrm{CHI}$ promoter}

Genomic DNA was extracted from four of the seven potato cultivars, Zhongshu 7, Favorita, Heijingang, and Désirée, using a Plant Genomic DNA kit (TIANGEN Biotech, Beijing, China).Two potato $\mathrm{CH} /$ promoter (PCP) sequences were amplified from each of the four cultivars using promoterspecific primers, CHIP1-F, CHIP2-F, and CHIP1-R (Table 1), designed according to the potato $\mathrm{CHI}$ gene (accession No. HQ659497) and potato genome data (http://solgenomics.net/). CHIP1-F and CHIP1-R were used to amplify a 1425 bp promoter fragment upstream of the $\mathrm{CHI}$ initiation codon ATG in each of the four cultivars, and CHIP2-F and CHIP1-R were employed to amplify a $665 \mathrm{bp}$ promoter fragment upstream of the $\mathrm{CHI}$ initiation codon in the Favorita cultivar. The PCR conditions were as follows: $94^{\circ} \mathrm{C}$ for $5 \mathrm{~min}$, followed by 35 cycles of $94^{\circ} \mathrm{C}$ for $45 \mathrm{~s}, 58^{\circ} \mathrm{C}$ for $30 \mathrm{~s}$, and $72^{\circ} \mathrm{C}$ for $45 \mathrm{~s}$, with a final extension step at $72^{\circ} \mathrm{C}$ for $10 \mathrm{~min}$. PCR products were then cloned into the pMD$19 \mathrm{~T}$ vector (TaKaRa Biotech, Dalian, China) for sequencing (Beijing Genomics Institute, China).

Table 1. Sequences of oligonucleotide primers used to analyze expression of the chalcone isomerase gene $(\mathrm{CHI})$ in potato and to amplify two $\mathrm{CHI}$ promoter sequences.

\begin{tabular}{llr}
\hline Primer & Sequence (5'-3') & Amplified fragment (bp) \\
\hline CHI-F & TGCCTATAAACAATAATCAGTG & 1152 \\
CHI-R & ACCATGCTTTTATTGAGCTATT & 454 \\
18 S-F & TGCCAGTAGTCATATGCTTGTCTC & 1425 \\
18 S-R & AGCCCGGTATTGTTATTTATTGTC & \\
CHIP1-F & CGGGGTACCTAGATAGTCACATGCGAAT & 665 \\
CHIP1-R & CATGCCATGGTTTTACACTGATTATGGTTATAGGC \\
CHIP2-F & CGGGGTACCACTGGATGACAATATGTACTTACG & \\
\hline Underlined sequences indicate restriction enzyme sites $(K p n l$ and Ncol) engineered for construction of binary vectors.
\end{tabular}




\section{Sequence analysis}

The PCP sequences were analyzed for the presence of cis-regulatory sequence motifs and related transcriptional factor binding sites (TFBs) using the plant cis-acting regulatory element (PlantCARE) database (Lescot et al., 2002) and plant cis-acting regulatory DNA elements (PLACE) database (Higo et al., 1999). The neural network promoter prediction (NNPP) server (Reese, 2001) was used to predict the transcription start site (TSS). Nucleotide sequence comparisons were performed using NCBI BLAST tools (http://www.ncbi.nlm.nih.gov/). Multiple sequence alignment was conducted using DNAMAN software (version 5.2.2, Lynnon Biosoft, Canada).

\section{Transient expression assay}

StCHIP1 which obtained 1425 bp PCP isolated from Heijingang and StCHIP2 which obtained 665 bp PCP from Favorita. The two PCP fragments were inserted into the binary vector pCMBIA1304, using the restriction enzymes Kpnl and Ncol, in place of the CaMV 35S promoter upstream of the GFP-GUS fusion. The recombinant vectors, named p1304-StCHIP1 and p1304StCHIP2, were transformed into the Agrobacterium tumefaciens strain GV3101 using the freezethaw method ( Hofgen and Willmitzer , 1988). Onion epidermis and fresh potato chips (1 to $2 \mathrm{~mm}$ thick) from the Heijingang, Desiree, $H Q$, Favorita, and Hui-2 cultivars were placed on Murashige and Skoog (MS) solid medium at $25^{\circ} \mathrm{C}$ for $48 \mathrm{~h}$, after infiltration in a suspension solution containing the Agrobacterium cells (Murashige and Skoog, 1962).

GFP protein was visualized using a fluorescence microscope (Zeiss Axio Imager A1, Germany). A histochemical GUS assay was carried out as described previously (Jefferson et al., 1987). Samples were incubated in the GUS assay buffer at $37^{\circ} \mathrm{C}$ overnight, washed with $70 \%$ ethanol, and then photographed under a stereomicroscope (Zeiss Stemi 2000-C, Germany).

\section{RESULTS}

\section{Expression of $\mathrm{CHI}$ genes in different potato cultivars}

$\mathrm{CHI}$ has previously been cloned from potato cultivar tubers (Wei et al., 2012). In this study, the expression level of $\mathrm{CH}$ in the peeled flesh of seven potato cultivars was examined using a semi-quantitative RT-PCR assay. The results show that $\mathrm{CH}$ l was only expressed in cultivars with colored tuber flesh, Heijingang and Ziyun1, but was not expressed in the other cultivars (Zhongshu 7, Hui-2, Favorita, HQ, and Désirée; Figure 1).

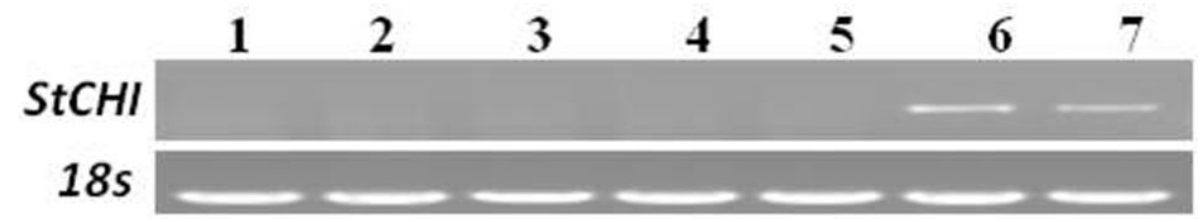

Figure 1. Semi-quantitative RT-PCR analysis of the anthocyanin structural gene StCHI in tuber flesh during tuber formation. Lane 1 = potato cultivar with yellow skin and white flesh (Zhongshu 7); lanes 2 and $3=$ cultivars with yellow skin and yellow flesh (Hui-2 and Favorita); lanes 4 and $5=$ cultivars with red skin and yellow flesh (HQ and Désirée); lanes 6 and $7=$ cultivars with purple skin and purple flesh (Ziyun1 and Heijingang). Expression of 18S rRNA was evaluated as an internal control. 


\section{Isolation and sequence analysis of PCP}

To determine why the $\mathrm{CHI}$ gene is silenced in the tissue of potato cultivars with noncolored flesh, a 1425 bp PCP sequence, including the CHI 5'-UTR, was isolated from four potato cultivars (Heijingang, Zhongshu 7, Favorita, and Désirée; Figure 2). The sequences were enriched in bases $\mathrm{A}$ and $\mathrm{T}(\sim 70 \%)$ along the whole sequence and contained a transcription start site (TSS) at the 1356th position. A total of $71 \mathrm{CREs}$, including core elements, such as the TATA-box and CAAT-box, anthocyanin gene CREs (30 copies), tissue-specific CREs (100 copies), environmental CREs (47 copies), and hormone-inducted CREs (16 copies), were isolated from the $\mathrm{CHI}$ promoter of the Heijingang cultivar (Table 2).

\section{Comparison of PCP CREs between potato and other plants}

To reveal the characteristics of the $\mathrm{CH}$ promoter among higher plants, we identified the $\mathrm{CHI}$ promoter region in tomato, tobacco, cacao, Arabidopsis, corn, petunia, barley, and rice using the GenBank and Solanaceae genomics databases (NM001247492, KJ730247, CM001882, CP002686, AC196033, X14589, AF474923, AF474922, respectively). There were 73 CREs in tomato, 75 CREs in tobacco, 74 CREs in cacao, 98 CREs in Arabidopsis, 67 CREs in corn, 83 CREs in petunia, 97 CREs in barley, 83 CREs in rice, and 71 CREs in potato, found by blasting the PLACE database, of which 19 CREs were common in the $\mathrm{CHI}$ promoters of all these plants (Figure 3). These CREs contained environmental CREs, such as dehydration-responsive CREs (CANNTG, WAACCA, and YAACKG) and light-responsive CREs (GATA, GRWAAW, and GATAA), tissue-specific CREs, such as root-specific CREs (ATATT), pollen-specific CREs (AGAAA and GTGA), seed-specific CREs (CAAT and RTTTTTR), and mesophyll-specific CREs (YACT and TAAAG). They also contained anthocyanin gene CREs (GRWAAW, CANNTG, and CNGTTR), which are the sites binding the MYB and MYC factors involved in tissue-specific anthocyanin biosynthesis genes, such as CHS and CHI (Solano et al., 1995; Stålberg et al., 1996; Hartmann et al., 2005), and TGACY connected with wounding. Compared with other plants assessed, potato has the largest number of the mesophyll-specific element YACT (35 copies) and the MYB binding site WAACCA ( 3 copies; Table 2). In addition to the 19 motifs, 2 specific motifs were only found in PCP, the GT-1 motif KWGTGRWAAWRW for light response and the DNA sequence TGTATATAT, which is over-represented in the light-repressed promoter (Table 2).

\section{Nucleotide variation in PCP among different potato cultivars}

Comparison of PCP nucleotide sequences among different potato cultivars was performed and the results are shown in Figure 2. Compared with the Heijingang cultivar, six nucleotide variation sites were detected in the PCP, located at the 162nd site ( $\mathrm{G}>\mathrm{A}$ in Désirée, Favorita, and Zhongshu 7 ), the 178th site ( $A>G$ in Favorita), the 573rd site ( $T>C$ in Favorita), the 1007th site ( $T>C$ in Zhongshu 7), the 989th site ( $A>G$ in Désirée), and the 1211st site ( $T>A$ in Désirée). These nucleotide variations caused the loss of several CREs in the non-pigmented-flesh cultivars Désirée, Favorite, and Zhongshu 7. For example, the G>A mutation at the 162nd site caused the loss of the mesophyll-specific element YACT and the MYB core element CNGTTR, connected with anthocyanin biosynthesis genes, in Désirée, Favorita, and Zhongshu 7; the $A>G$ mutation at the 178th position caused the loss of the light response element TGTATATAT in Favorita; the 
Sequence analysis of a chalcone isomerase gene promoter

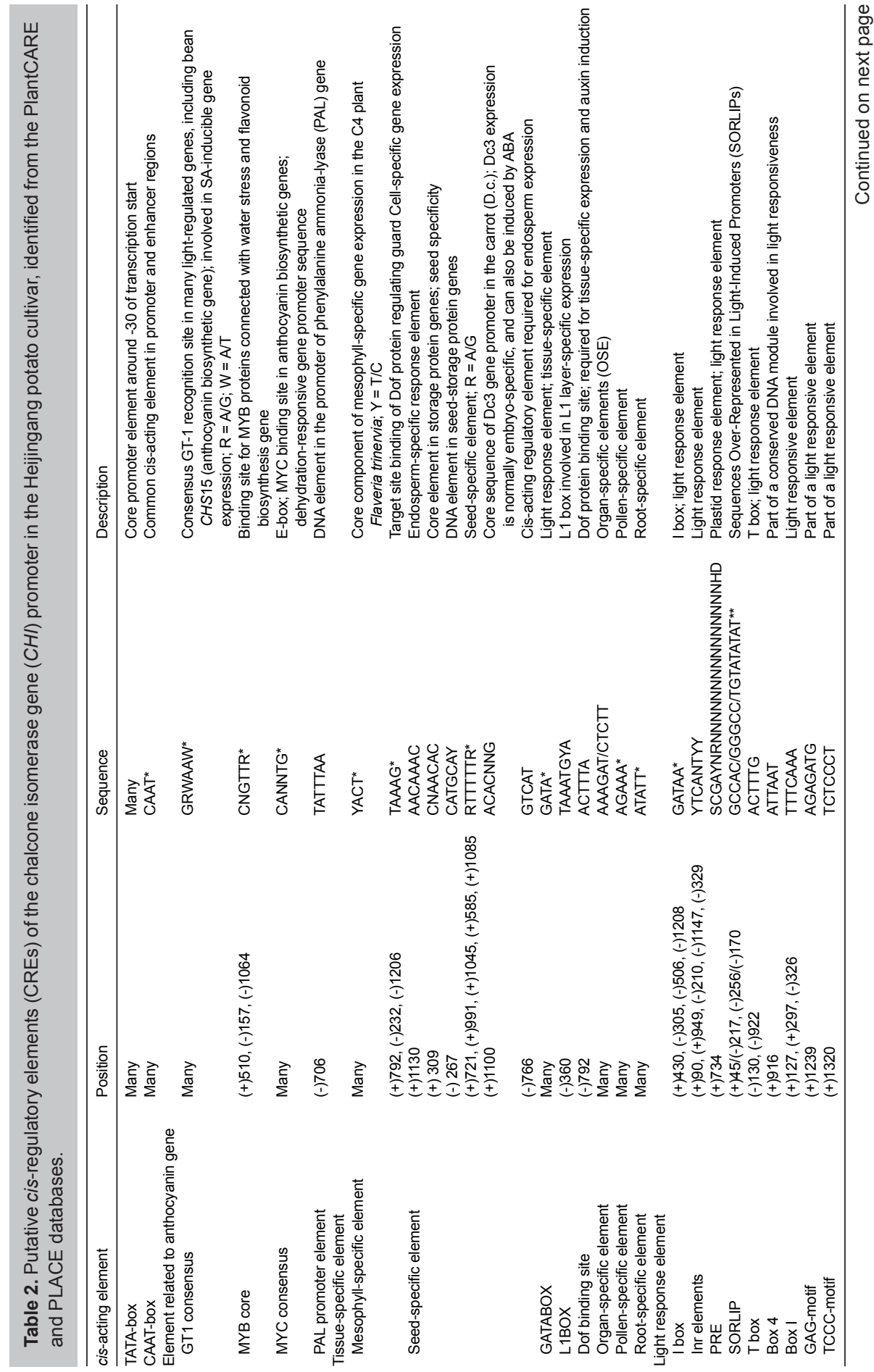




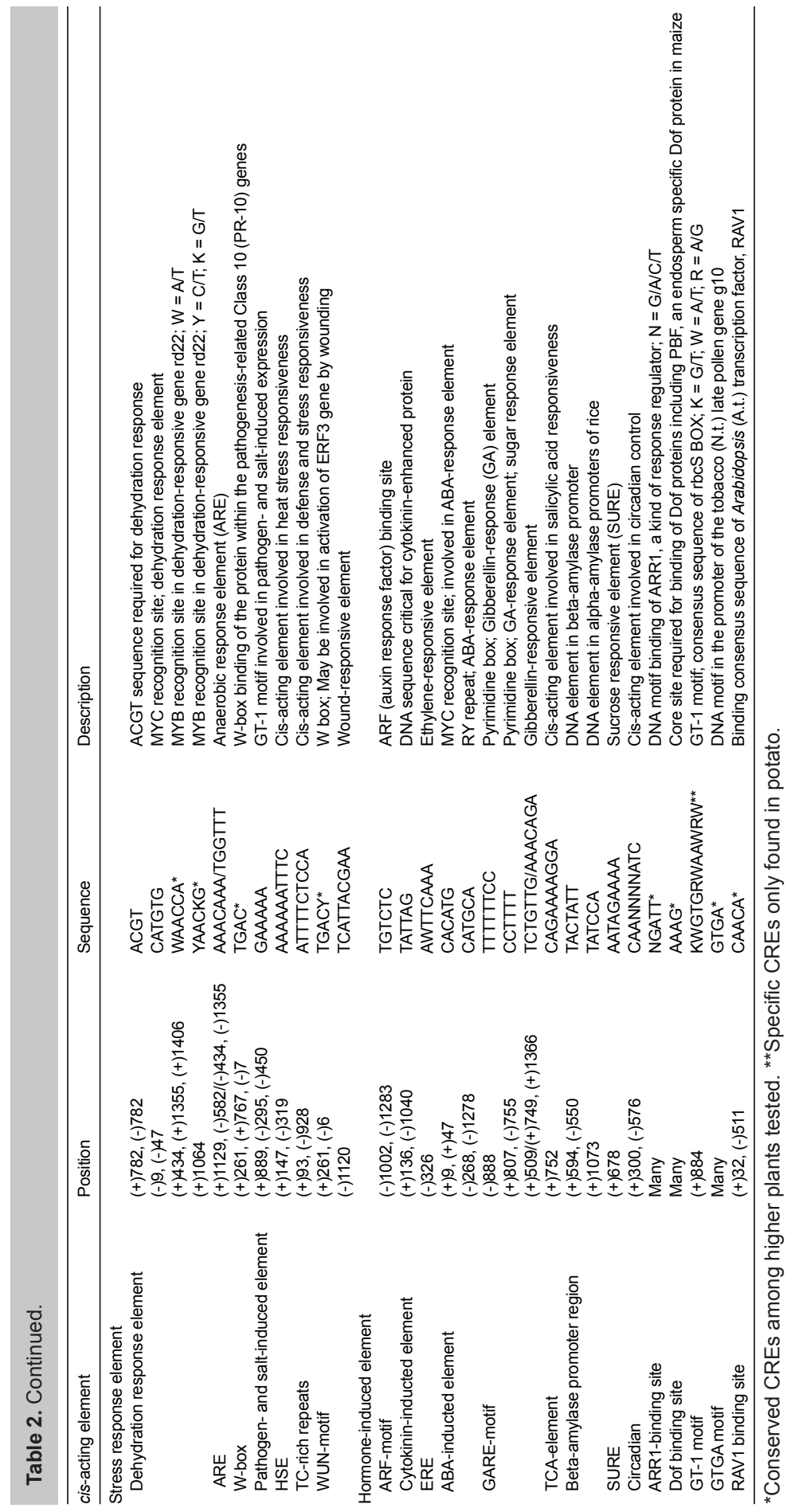


T>C mutation at the 573rd position caused the loss of NGATT in Favorita; the T>C mutation at the 1007th position caused the loss of the light response element GT-1 in Zhongshu 7; the $A>G$ mutation at the 989th position caused the loss of the root-specific motif ATATT in Désirée; and the T>A mutation at the 1211st position caused the loss of the GATA box, GRWAAW, and GATAA, connected with light and tissue specificity, in Désirée. In contrast, the G>A mutation at the 162nd position resulted in the Désirée, Favorita, and Zhongshu 7 cultivars gaining the CAAT box and the ATATT motifs (Figure 2). These results show that cultivars with higher levels of anthocyanins have more CREs related to tissue-specificity, light response, and anthocyanin biosynthesis.

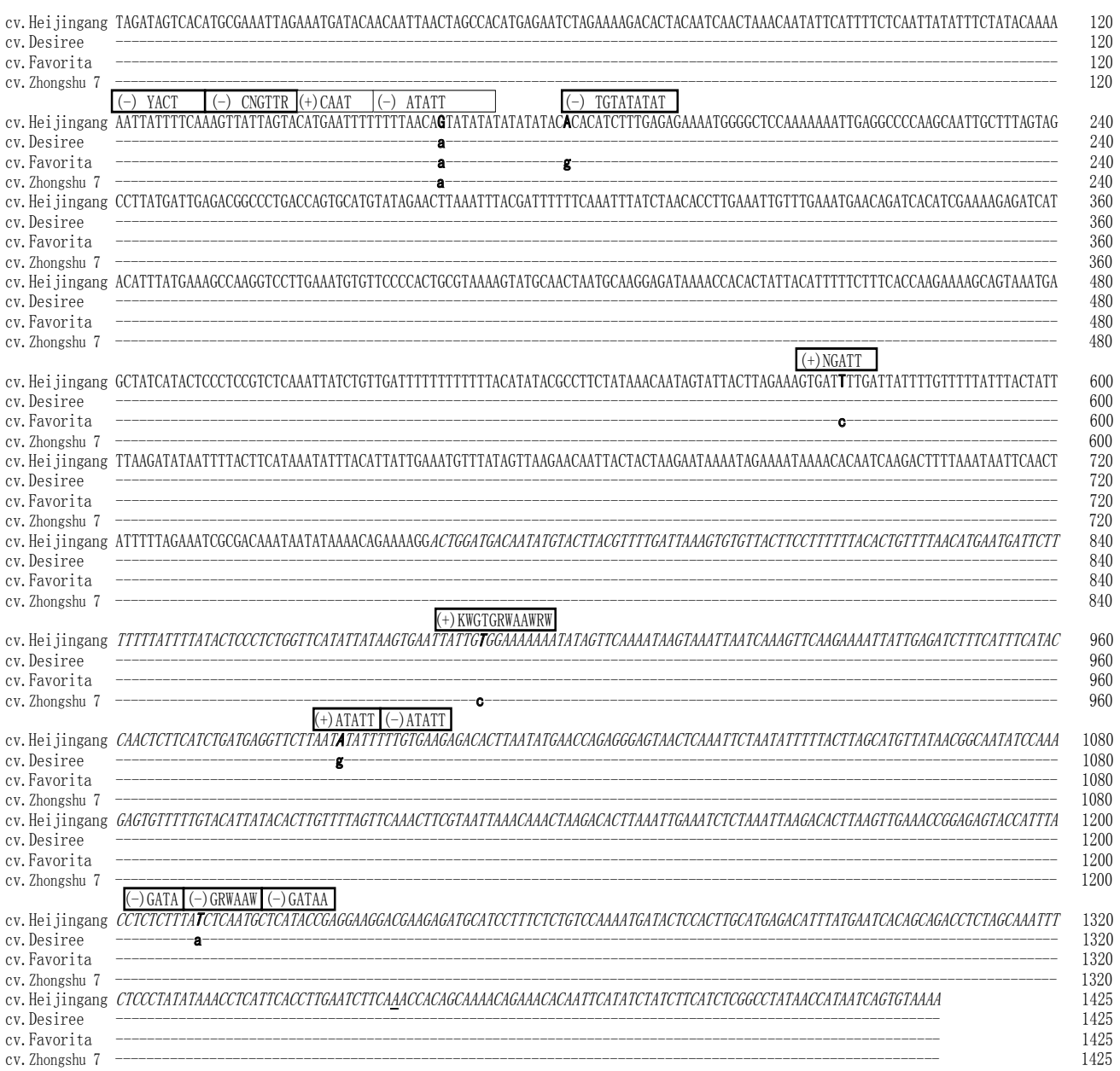

Figure 2. Sequence alignment of the chalcone isomerase gene $(\mathrm{CHI})$ promoter, StCHIP1, in different potato cultivars: Heijingang (purple skin and flesh), Désirée (red skin and yellow flesh), Zhongshu 7 (yellow skin and white flesh), and Favorita (yellow skin and flesh). The 665 bp sequence (StCHIP2) is italicized. Capital and small letters in bold type indicate single nucleotide differences in their respective positions. Corresponding motifs isolated from the PLACE database are shown in rectangular boxes. Compared with the Heijingang cultivars, cultivars with non-colored flesh have lost motifs (boxes with thick borders) and gained motifs (boxes with thin borders). Letters underlined denote putative transcription start sites (TSS). 


\section{Analysis of PCP activity}

Whether nucleotide variations in PCP influence promoter activity was investigated further. The two PCP sequences, StCHIP1 and StCHIP2, were inserted into the binary vector pCMBIA1304 in place of the CaMV35S fragment upstream of GFP and GUS, respectively. The constructs, p1304-StCHIP1 and p1304-StCHIP2, were then introduced into onion epidermal cells and potato tuber flesh using an Agrobacterium-mediated method (Figure 4). Cytological observation showed that the green fluorescence signal was present in onion cells (Figure 5). X-gluc staining revealed that the GUS gene was expressed under the direction of StCHIP1 and StCHIP1 in the cultivars Heijingang, Désirée, HQ, Favorita, and Hui-2 (Figure 5). These results suggest that the single nucleotide variations in PCP were not the primary cause of the undetected expression levels of the $\mathrm{CHI}$ gene in potato cultivars with non-colored flesh.

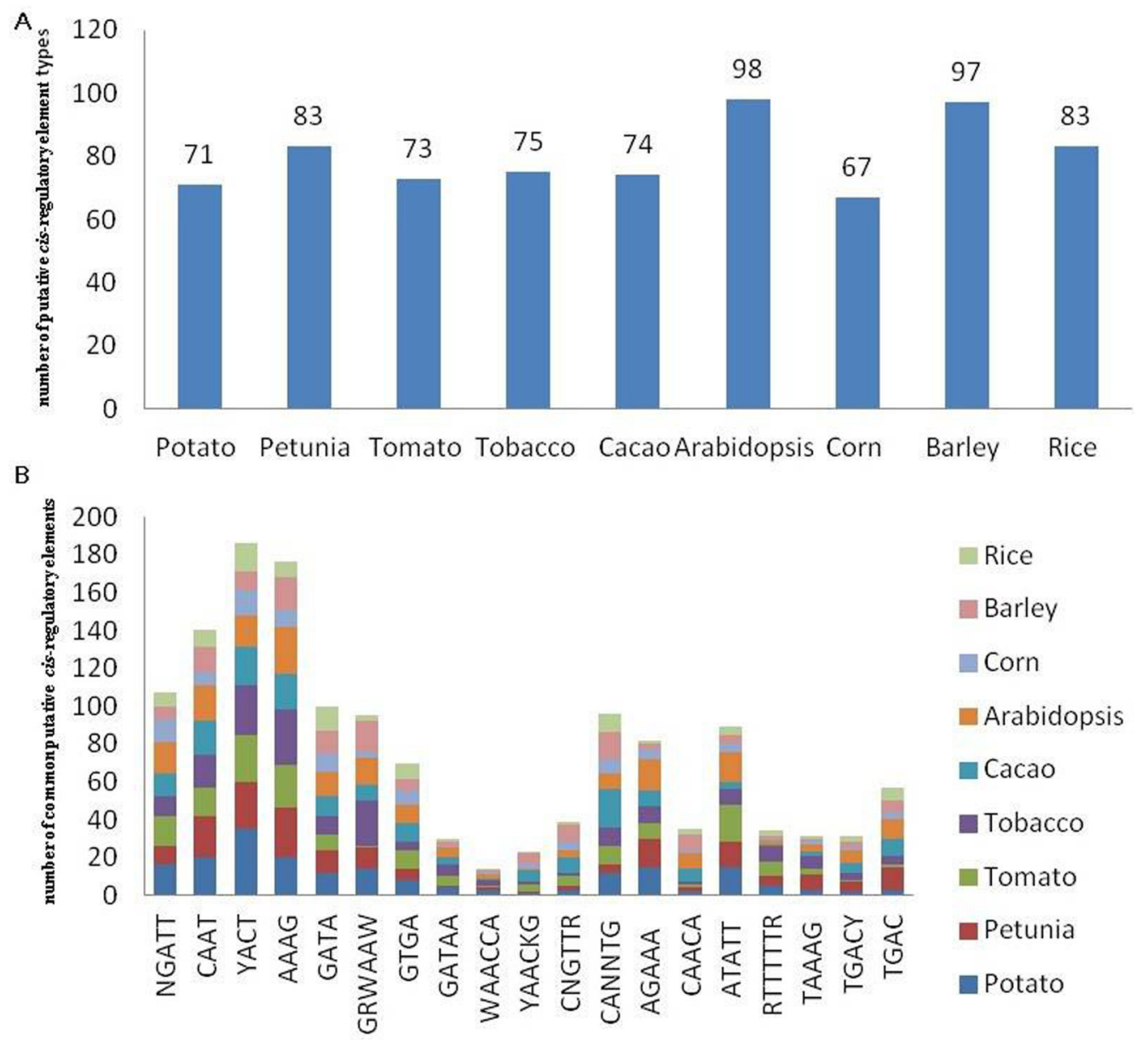

Figure 3. Putative cis-regulatory elements found in chalcone isomerase gene $(\mathrm{CHI})$ promoters in 9 higher plants. (A) total number of putative cis-regulatory element types, (B) Occurrence of 19 common cis-regulatory elements. 

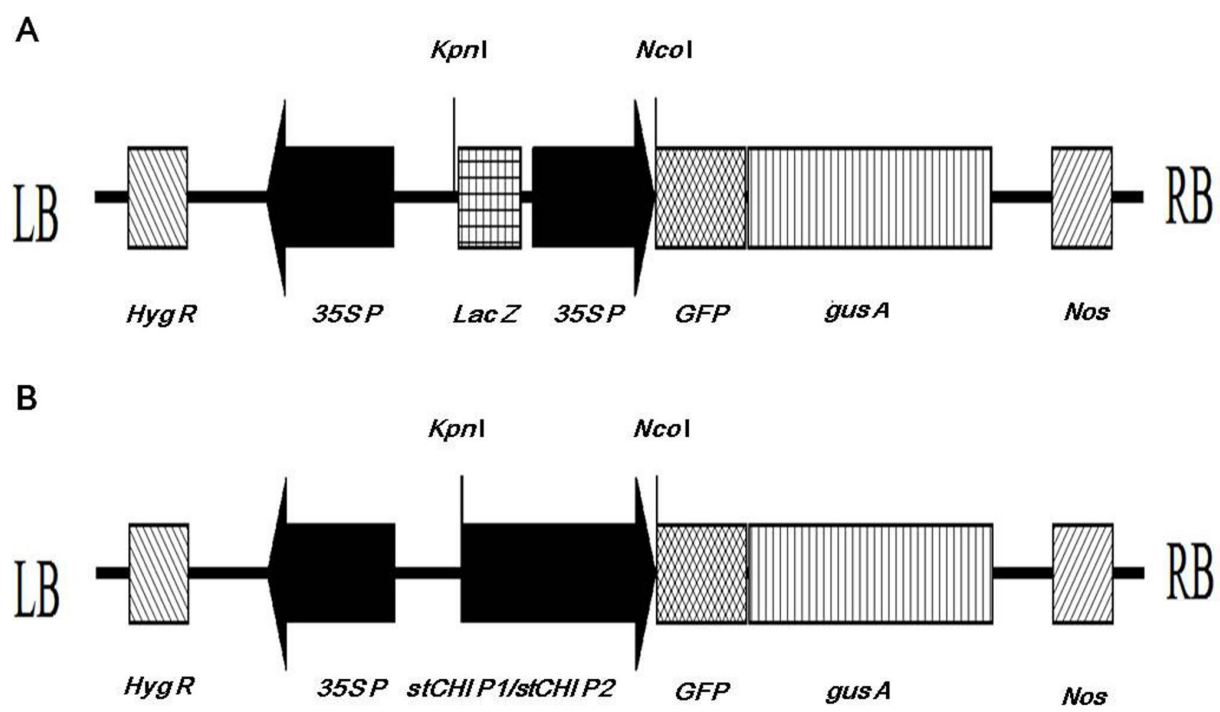

Figure 4. Schematic representation of the T-DNA region of the binary vector. The 1425 and 665 bp chalcone isomerase gene $(\mathrm{CHI})$ promoter fragments amplified from the Heijingang cultivar were used to replace the $35 \mathrm{~S}$ promoter upstream of the GFP gene in pCAMBIA1304 (A), and the recombinant vectors were named p1304-StCHIP1 and p1304-StCHIP2, respectively (B). LB, T-DNA left border; Hyg R, hygromycin (R); 35S P, CaMV 35 S promoter; lacZ, lacZ lapha; GFP, green fluorescent protein gene; gusA, $\beta$-Glucuronidase gene; nos, Nos poly-A; RB, T-DNA right border.

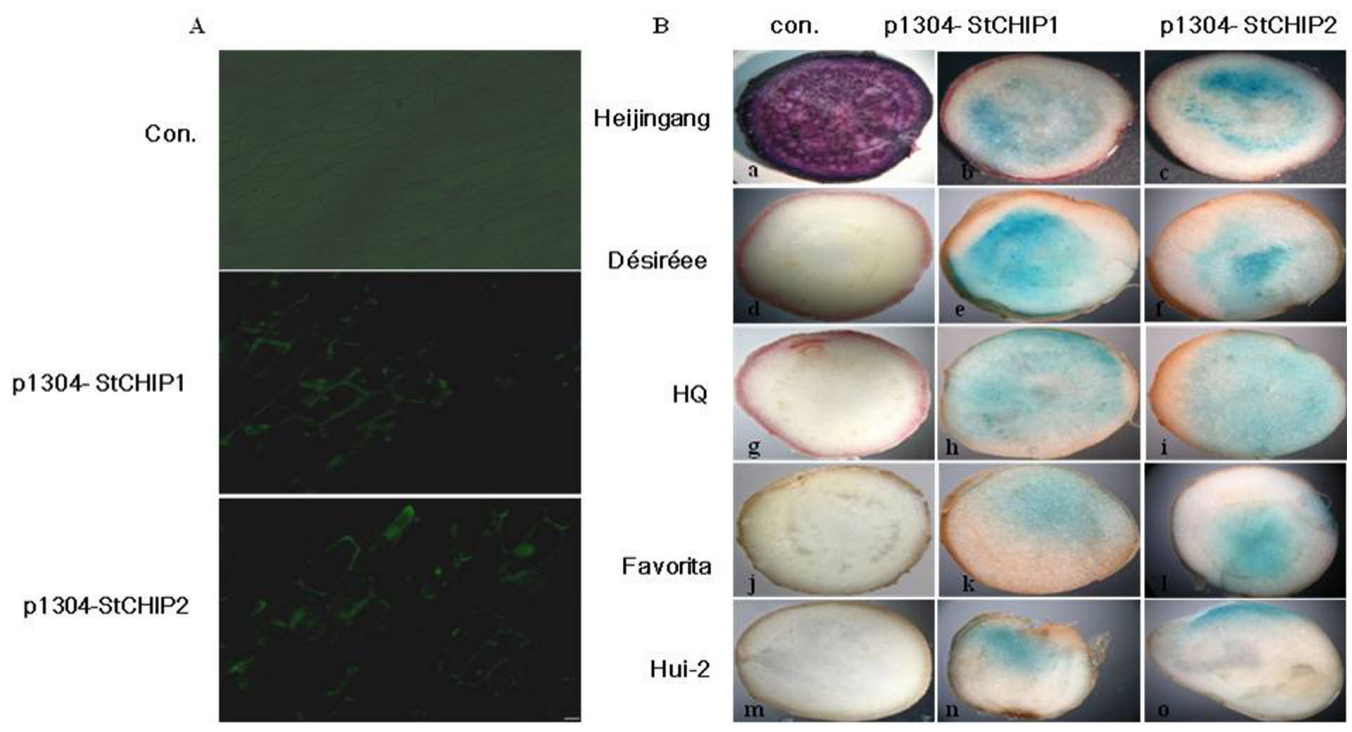

Figure 5. Transient expression of a green fluorescent protein gene (GFP) and a $\beta$-glucuronidase gene (GUS) driven by potato chalcone isomerase gene $(\mathrm{CHI})$ promoters. (A) GFP expression in epidermal onion cells is driven by the vectors p1304-StCHIP1 and p1304-SCHIP2. Con, denotes non-tested onion epidermis control. (B) GUS expression in pigmented and non-pigmented potato tuber flesh. Panels a, d, g, j, and m corresponded to non-tested (control) potato flesh; panels b, e, h, k, and n corresponded to GUS expression driven by p1304-StCHIP1; and panels c, f, i, I, and o corresponded to GUS expression driven by p1304-StCHIP2. 


\section{DISCUSSION}

The promoter is an essential regulatory element of gene expression. In the past, the $\mathrm{CHI}$ gene has been isolated from many plants, but its promoter has only been reported in $P$. hybrida, where $\mathrm{CH}$ l promoter sequences were isolated and the cis-element 'anther box' was discovered, revealing high homology among flavonoid gene promoters (van Tunen et al., 1989). In this study, PCP was cloned from 4 different potato cultivars (Heijingang, Zhongshu 7, Désirée, and Favorita; Figure 2). Sequence analysis showed that PCP exhibits high identity $(\sim 100 \%)$ among potato cultivars but low identity ( 39 to $60 \%$ ) among higher plants tested (data not shown), indicating that $\mathrm{PCP}$ is conserved in potato cultivars but variant among higher plants. To the best of our knowledge, similar studies investigating PCP have not been conducted; related research was about $\mathrm{CHI}$ gene observed to have low similarity in different dicot groups ( 42 to $65 \%)$, except for the closely related legumes Medicago sativa and Phaseolus vulgaris ( 80\%; Druka et al., 2003).

In general, plant promoters consist of two types of elements: core elements and regulatory elements. The former influence promoter activity and the latter determine gene function. $\mathrm{CHI}$ is an important enzyme in anthocyanin biosynthesis and is often induced by environmental conditions (McKhann and Hirsch, 1994; Fofana et al., 2002; Pandey et al., 2014), thus, the CHI promoter inevitably possesses corresponding functional elements in its sequence structure. In this study, we observed that, aside from the core elements TATA-box and CAAT-box, there are several functional CREs in potato, which are related to tissue specificity, light response, stress, anthocyanin structural genes, etc.

Anthocyanin structural genes are uniformly controlled by special transcription factors in the plant metabolic pathway (Bovy et al., 2002; Jung et al., 2009), among which highly conserved tissue-specific motifs are isolated (van Tunen et al., 1989; Hartmann et al., 2005). Here, we isolated four important CREs connected with anthocyanin synthesis genes, including the GT-1 consensus found in the bean CHS15 promoter, the phenylalanine ammonia-lyase (PAL) promoter sequence found in the promoter of the PAL gene, which is upstream of $\mathrm{CHI}$ in the flavonoid biosynthetic pathway, the MYC consensus (E-box), which is the MYC recognition motif known in the anthocyanin gene promoter, and the MYB core found in the promoter of the Petunia CHSJ gene and bond of MYB.Ph3 involved in regulation of flavonoid biosynthesis (Solano et al., 1995). These results suggest that $\mathrm{CHI}$ and other anthocyanin structural genes may be regulated by common transcription factors, such as MYB and MYC proteins, in potato (Table 2).

MYB- and MYC-type proteins are important regulatory factors in regulating anthocyanin structural genes during tissue-specific accumulation of anthocyanins (Bovy et al., 2002; Shimizu et al., 2011; Pandey et al., 2014). The MYB transcription factor encoded by AN2 from potato influences pigment accumulation in the tubers (Jung et al., 2009). Anthocyanin accumulation is affected by many factors, including stress and light, and by controlling regulatory factors, such as MYB proteins (Procissi et al., 1997; Fofana et al., 2002). In a previous study, the CHS promoter showed activity under control of the MYB factor in Arabidopsis thaliana under light (Hartmann et al., 2005). Here, several putative motifs for defense were isolated in PCP, including two tissuespecific anthocyanin gene elements, MYB core for water stress and MYC consensus (E-box) for dehydration. Many other important light-responsive motifs were also found such as GATA box and the GT1 consensus in high occurrence (Figure 3). The environmental motifs required for light signal activation and stress may influence $\mathrm{CHI}$ expression by binding to anthocyanin regulatory factors.

Comparison of CREs among higher plants tested revealed 19 conserved CREs, consisting 
of W-box, E-box, I-box, MYB, and MYC motifs involved in flavonoid synthesis, tissue specificity, light response, hormone response, and defense response (Figure 3). Interestingly, several CREs were mainly found in the Solanaceae, including potato, and were related to the promoter of the PAL gene, L1 layer-specific expression, seed-specific motif RY repeats, MYC recognition sites, dehydration response elements, ABA elements, GA elements, etc. (data not shown). In PCP, two light response motifs, KWGTGRWAAWRW and TGTATATAT, were found to only exist in the potato $\mathrm{CHI}$ promoter (Table 2). Therefore, PCP has retained several conserved sequence motifs of the $\mathrm{CHI}$ gene and produced its own specific motifs during its evolutionary history.

In biological evolution, nucleotide evolution caused by natural mutation can occur at any position on the DNA, and this mutation may be significant in biology (Druka et al., 2003). In previous studies, the eukaryotic promoter was changed by modifying 1 to 2 bases in the binding site, which affects gene expression levels, and proanthocyanidin-free barley mutants showed drastically reduced flavonoid levels compared to the wide-type control following single base changes in $\mathrm{CHI}$, demonstrating that single nucleotide variations can alter gene expression (Druka et al., 2003; Rajkumar et al., 2013). In potato, tubers with different colored flesh result from anthocyanin accumulation. In order to determine the role of PCP in anthocyanin accumulation, we analyzed simple nucleotide variation in PCP in different potato cultivars. Nucleotide variation was evident at 6 sites in PCP, resulting in changes in CREs (Figure 2), similar to the variability in the 5'-UTR region of $\mathrm{CH}$ represented by single nucleotide polymorphisms in the common bean (McClean and Lee, 2007). However, these single nucleotide variations did not appear to influence promoter activity, based on our PCP transient expression results (Figure 5). From a promoter perspective, this result revealed that PCP was not a decisive factor in anthocyanin metabolism in potato cultivars with noncolored tubers and silence of $\mathrm{CHI}$ gene expression may be attributed to special regulatory factors.

In conclusion, we isolated the potato $\mathrm{CH}$ / promoter from potato cultivars and analyzed its structural characteristics and differences in CREs between potato and other higher plants. We also assessed variation in PCP among different potato cultivars, revealing that PCP may be involved in growth and development, anthocyanin biosynthesis, and stress response, etc. However, we determined that single nucleotide variations in PCP were not responsible for silencing the $\mathrm{CHI}$ gene in the tested potato cultivars with non-colored flesh. These results may be helpful in furthering our understanding of PCP evolution and transcriptional regulation.

\section{ACKNOWLEDGMENTS}

Research supported by grants from the National Natural Science Foundation of China (\#11171155), the National Pear Industry Technology System (\#CARS-29), and a project funded by the Priority Academic Program Development of Jiangsu Higher Education Institutions: Modern Horticultural Science (PAPD).

\section{REFERENCES}

Bieza K and Lois R (2001). An Arabidopsis mutant tolerant to lethal ultraviolet-B levels shows constitutively elevated accumulation of flavonoids and other phenolics. Plant Physiol. 126: 1105-1115.

Bovy A, de Vos R, Kemper M, Schijlen E, et al. (2002). High-flavonol tomatoes resulting from the heterologous expression of the maize transcription factor genes LC and C1. Plant Cell. 14: 2509-2526.

Cai H, Marczylo TH, Teller N, Brown K, et al. (2010). Anthocyanin-rich red grape extract impedes adenoma development in the Apc(Min) mouse: pharmacodynamic changes and anthocyanin levels in the murine biophase. Eur. J. Cancer 46: 811-817. 
Druka A, Kudrna D, Rostoks N, Brueggeman R, et al. (2003). Chalcone isomerase gene from rice (Oryza sativa) and barley (Hordeum vulgare): physical, genetic and mutation mapping. Gene 302: 171-178.

Eichhorn S and Winterhalter P (2005). Anthocyanins from pigmented potato (Solanum tuberosum L.) varieties. Food Res. Int. 38: 943-948.

Fofana B, McNally DJ, Labbé C, Boulanger R, et al. (2002). Milsana-induced resistance in powdery mildew-infected cucumber plants correlates with the induction of chalcone synthase and chalcone isomerase. Physiol. Mol. Plant Pathol. 61: 121132.

Grotewold E and Peterson T (1994). Isolation and characterization of a maize gene encoding chalcone flavonone isomerase. Mol. Gen. Genet. 242: 1-8.

Hartmann U, Sagasser M, Mehrtens F, Stracke R, et al. (2005). Differential combinatorial interactions of cis-acting elements recognized by R2R3-MYB, BZIP, and BHLH factors control light-responsive and tissue-specific activation of phenylpropanoid biosynthesis genes. Plant Mol. Biol. 57: 155-171.

Higo K, Ugawa Y, Iwamoto M and Korenaga T (1999). Plant cis-acting regulatory DNA elements (PLACE) database: 1999. Nucleic Acids Res. 27: 297-300.

Hofgen R and Willmitzer L (1988). Storage of competent cells for Agrobacterium transformation. Nucleic Acids Res. 16: 9877.

Hong L, Qian Q, Tang D, Wang KJ, et al. (2012). A mutation in the rice chalcone isomerase gene causes the golden hull and internode 1 phenotype. Planta 236: 141-151.

Hwang YP, Choi JH, Choi JM, Chung YC, et al. (2011). Protective mechanisms of anthocyanins from purple sweet potato against tert-butyl hydroperoxide-induced hepatotoxicity. Food Chem. Toxicol. 49: 2081-2089.

Jefferson RA, Kavanagh TA and Bevan MW (1987). GUS fusions: beta-glucuronidase as a sensitive and versatile gene fusion marker in higher plants. EMBO J. 6: 3901-3907.

Jung CS, Griffiths HM, De Jong DM, Cheng S, et al. (2009). The potato developer (D) locus encodes an R2R3 MYB transcription factor that regulates expression of multiple anthocyanin structural genes in tuber skin. Theor. Appl. Genet. 120: 45-57.

Koes R, Verweij W and Quattrocchio F (2005). Flavonoids: a colorful model for the regulation and evolution of biochemical pathways. Trends Plant Sci. 10: 236-242.

Lescot M, Déhais P, Thijs G, Marchal K, et al. (2002). PlantCARE, a database of plant cis-acting regulatory elements and a portal to tools for in silico analysis of promoter sequences. Nucleic Acids Res. 30: 325-327.

Li F, Jin Z, Qu W, Zhao D, et al. (2006). Cloning of a cDNA encoding the Saussurea medusa chalcone isomerase and its expression in transgenic tobacco. Plant Physiol. Biochem. 44: 455-461.

Liu Y, Zhao S, Wang J, Zhao C, et al. (2015). Molecular cloning, expression, and evolution analysis of type II CHI gene from peanut (Arachis hypogaea L.). Dev Genes Evol. 225: 1-10.

Lu QN and Yang Q (2006). cDNA cloning and expression of anthocyanin biosynthetic genes in wild potato (Solanum pinnatisectum). Afr. J. Biotechnol. 5: 811-818.

McClean PE and Lee RK (2007). Genetic architecture of chalcone isomerase non-coding regions in common bean (Phaseolus vulgaris L.). Genome 50: 203-214.

McKhann HI and Hirsch AM (1994). Isolation of chalcone synthase and chalcone isomerase cDNAs from alfalfa (Medicago sativa L.): highest transcript levels occur in young roots and root tips. Plant Mol. Biol. 24: 767-777.

Mehdy MC and Lamb C J (1987). Chalcone isomerase cDNA cloning and mRNA induction by fungal elicitor, wounding and infection. EMBO J. 6: 1527-1533.

Murashige T and Skoog F (1962). A revised medium for rapid growth and bio assays with tobacco tissue cultures. Physiol. Plant. 15: 473-497.

Naito K, Umemura Y, Mori M, Sumida T, et al. (1998). Acylated pelargonidin glycosides from a red potato. Phytochemistry 47: 109-112.

Netzel M, Netzel G, Kammerer DR, Schieber A, et al. (2007). Cancer cell antiproliferation activity and metabolism of black carrot anthocyanins. Innov. Food Sci. Emerg. Technol. 8: 365-372.

Nishihara M, Nakatsuka T and Yamamura S (2005). Flavonoid components and flower color change in transgenic tobacco plants by suppression of chalcone isomerase gene. FEBS Lett. 579: 6074-6078.

Pandey A, Misra P, Bhambhani S, Bhatia C, et al. (2014). Expression of Arabidopsis MYB transcription factor, AtMYB111, in tobacco requires light to modulate flavonol content. Sci. Rep. 4: 5018.

Procissi A, Dolfini S, Ronchi A and Tonelli C (1997). Light-dependent spatial and temporal expression of pigment regulatory genes in developing maize seeds. Plant Cell 9: 1547-1557.

Rajkumar AS, Dénervaud N and Maerkl SJ (2013). Mapping the fine structure of a eukaryotic promoter input-output function. Nat. Genet. 45: 1207-1215.

Reese MG (2001). Application of a time-delay neural network to promoter annotation in the Drosophila melanogaster genome. Comput. Chem. 26: 51-56. 
Rodriguez-Saona LE, Giusti MM and Wrolstad RE (1998). Anthocyanin pigment composition of red-fleshed potatoes. J. Food Sci. 63: 458-465.

Muir SR, Collins GJ, Robinson S, Hughes S, et al. (2001). Overexpression of petunia chalcone isomerase in tomato results in fruit containing increased levels of flavonols. Nat. Biotechnol. 19: 470-474.

Shimizu Y, Maeda K, Kato M and Shimomura K (2011). Co-expression of GbMYB1 and GbMYC1 induces anthocyanin accumulation in roots of cultured Gynura bicolor DC. plantlet on methyl jasmonate treatment. Plant Physiol. Biochem. 49: 159-167.

Solano R, Nieto C, Avila J, Cañas L, et al. (1995). Dual DNA binding specificity of a petal epidermis-specific MYB transcription factor (MYB.Ph3) from Petunia hybrida. EMBO J. 14: 1773-1784.

Stålberg K, Ellerstöm M, Ezcurra I, Ablov S, et al. (1996). Disruption of an overlapping E-box/ABRE motif abolished high transcription of the napA storage-protein promoter in transgenic Brassica napus seeds. Planta 199: 515-519.

van Tunen AJ, Koes RE, Spelt CE, van der Krol AR, et al. (1988) Cloning of the two chalcone flavanone isomerase genes from Petunia hybrida: coordinate, light-regulated and differential expression of flavonoid genes. EMBO J. 7: $1257-1263$.

van Tunen AJ, Hartman SA, Mur LA and Mol JN (1989). Regulation of chalcone flavanone isomerase (CHI) gene expression in Petunia hybrida: the use of alternative promoters in corolla, anthers and pollen. Plant Mol. Biol. 12: 539-551.

Wang B, Wang QY, Yin JM, Chen M, et al. (2011). Molecular cloning and expression analysis of an ANS homologous gene from Solanum cardiphyllum. Plant Physiol. J. 47: 1103-1108.

Wang Y, Li J and Xia R (2010). Expression of chalcone synthase and chalcone isomerase genes and accumulation of corresponding flavonoids during fruit maturation of Guoqing No. 4 satsuma mandarin (Citrus unshiu Marcow). Sci Hortic. 125: 110-116.

Wei Q, Wang QY, Feng ZH, Wang B, et al. (2012). Increased accumulation of anthocyanins in transgenic potato tubers by overexpressing the 3GT gene. Plant Biotechnol. Rep. 6: 69-75. 